\title{
Néolithisation atlasique pastorale et pré-agricole en Algérie : comportements de subsistance
}

Colette Roubet

\section{OpenEdition}

\section{Journals}

\section{Édition électronique}

URL : https://journals.openedition.org/encyclopedieberbere/2725

DOI : 10.4000/encyclopedieberbere.2725

ISSN : 2262-7197

Éditeur

Peeters Publishers

\section{Édition imprimée}

Date de publication : 5 octobre 2012

Pagination : 5489-5496

ISBN : 978-90-429-2718-6

ISSN : 1015-7344

Référence électronique

Colette Roubet, « Néolithisation atlasique pastorale et pré-agricole en Algérie : comportements de subsistance », Encyclopédie berbère [En ligne], 34 | 2012, document N45, mis en ligne le 15 décembre 2020, consulté le 17 février 2022. URL : http://journals.openedition.org/encyclopedieberbere/2725 ; DOI : https://doi.org/10.4000/encyclopedieberbere.2725

Ce document a été généré automatiquement le 17 février 2022.

(c) Tous droits réservés 


\title{
Néolithisation atlasique pastorale et pré-agricole en Algérie : comportements de subsistance
}

\author{
Colette Roubet
}

1 Durant l'Holocène lorsque les premiers pasteurs choisissent d'investir le territoire atlasique, ils enclenchent par cet acte initial un nouveau mode de fonctionnement, ouvert et interactif : la Néolithisation. La Néolithisation va s'imposer comme étant une rupture comportementale et territoriale décisive avec les principaux repères antérieurs pour conduire un processus continu installant de nouveaux fondements économiques et sociétaux. On a souhaité le traduire en créant une entité nouvelle dite Néolithisation atlasique pastorale et pré-agricole (Roubet 2003, 2006), générique mais restrictive, accueillant toute spécificité culturelle.

2 En Algérie, le premier noyau du pastoralisme atlasique actuellement identifié s'est installé depuis le VIII ${ }^{\mathrm{e}}$ jusqu'au VI ${ }^{\mathrm{e}}$ millénaire cal BP. Sur ce territoire d'altitude resté peu ou pas fréquenté, aucun signe d'une agriculture débutante n'a été identifié durant l'Holocène ancien-moyen. Le faciès culturel qui l'a instauré est le Néolithique de tradition capsienne s. s. (Roubet 1979). Durant cette période, ni ce pastoralisme ni ce faciès culturel ne sont restés dissociés, figés ou repliés, tous deux se sont inscrits dans un engrenage, une coévolution dynamique, ininterrompue.

Plus tard, durant les temps historiques, lorsque sous de nouvelles formes et d'autres types d'économies ce pastoralisme initial, atlasique, a été transmis, il s'est encore développé, conservant aux communautés montagnardes berbères cette spécificité déterminante.

4 Ainsi, inauguré par la néolithisation, le fait pastoral, majeur et fédérateur, n'a pas enraciné que des constantes socio-économiques : au fil du temps il est parvenu à créer entre les hommes, leur bétail et leur territoire montagnard, un état de symbiose pérennisé. Les arguments qui le soutiennent s'appuient sur une présence ininterrompue de l'habitat pastoral en altitude et sur une grande familiarité des communautés avec leurs lieux de séjour. On en perçoit aujourd'hui des signes à travers 
l'intime attachement dont témoignent la valorisation de l'espace montagnard et l'harmonieuse insertion des transformations anthropiques dans ce "terroir ", nouvel espace domestiqué et organisé durant l'Holocène. Ces conditions qui longtemps survécurent se modifient aujourd'hui. En effet les sociétés modernes placées devant de nouvelles contraintes optent pour d'autres activités contribuant aux déséquilibres des paysages et à l'amoindrissement des ressources.

5 Le territoire atlasique de l'Algérie qui s'étend des Némencha* au Monts des Ksour ne saurait être tronqué de ses développements structuraux orientaux (Tunisie) et occidentaux (Maroc). L'inégal avancement des recherches sur l'adoption de la domestication animale et sur l'élevage conduit à isoler provisoirement aussi l'Algérie atlasique, de l'Algérie tellienne et saharienne. Nous examinerons ici les comportements de subsistance à partir d'exemples complémentaires: les uns issus de témoins alimentaires, les autres provenant des représentatations graphiques.

\section{Comportements de subsistance : la domestication animale et l'élevage en Algérie atlasique, le statut social des bergers}

6 Grottes et abris des massifs mitoyens orientaux de l'Aurès et des Némencha occupés par des pasteurs depuis l'Holocène moyen ( $\mathrm{VI}^{\mathrm{e}}$ millénaire $\mathrm{BC}$ ) ont livré les informations de référence (Roubet, EB XXVII, p. 4205-4228, et N38 "Némencha»). Leurs contextes culturels ayant déjà fait l'objet de présentations (Roubet 1968, 1979), seuls les comportements de subsistance seront ici retenus.

\section{- Massif de l'Aurès}

7 La grotte Capéletti du Khanguet Si Mohamed Tahar* a fourni les données chronologiques calibrées (Prog. Cal 98) qui inscrivent les comportements d'une production de la subsistance entre 7855 et 4414 cal BP soit 5906 et 2465 cal BC, au cours de quatre séries d'occupation, dont la plus ancienne est la première (Tableau 1). Elevage, chasses et collectes ont assuré l'alimentation des pasteurs en montagne. Moutons, chèvres et bovins domestiques représentent l'alimentation substantielle. S'y ajoute le produit de deux catégories de chasses, l'une au sanglier*, en montagne (Suinés), l'autre aux antilopes, sur les piémonts (Antilopinés et Oryginés). Les données quantitatives ne doivent pas faire illusion, le nombre d'individus consommés reste plutôt faible en grotte. Mais qu'en a-t-il été au dehors, sur les alpages, durant les trajets et les séjours ailleurs? De la première série d'occupation jusqu'à la quatrième, la consommation a été estimée (parties anatomiques identifiées) pour les ovins-caprins, à $4,20,30$ et 4 individus et, pour les bovins à un animal, six, trois, puis un animal enfin. Durant les $2 \mathrm{e}$ et 3e séries d'occupations, un accroissement de la consommation a été observé, sans que les pasteurs fassent souvent appel à l'espèce sauvage locale, le sanglier, pourtant bien implanté dans ces biotopes (forêts de chênes yeuses, ballotes, Quercus ilex var. Ballota Desfontaines), comme si cet appoint carné n'avait résulté que d'un acte opportuniste de gourmet. Quant aux diverses captures sur les piémonts, elles n'ont procuré que gazelles et antilopes, lièvres, hérissons, tortues, batraciens et oiseaux. Du moins est-ce l'image que nous laissent entrevoir leurs ossements rejetés en grotte, au retour des transhumances. Ajoutons l'apport d'importantes collectes de 
gastéropodes terrestres. Les hélicidés furent préparés en grotte durant les $2^{\mathrm{e}}$ et $3^{\mathrm{e}}$ séries d'occupations, périodes de nourritures abondantes et variées, en relation avec un éventuel accroissement du groupe humain et surtout avec d'excellentes conditions climatiques.

\begin{tabular}{|c|c|c|c|c|c|c|c|c|c|}
\hline \multirow[t]{3}{*}{ DETERMINATION } & \multicolumn{8}{|c|}{ GROTTE CAPELETTI : SERIES D'OCCUPATION NEOLITHIQUES } & \multirow[t]{3}{*}{ TOTAL } \\
\hline & \multicolumn{2}{|l|}{$1^{\text {ère }}$} & \multicolumn{2}{|l|}{$2^{\mathrm{e}}$} & \multicolumn{2}{|l|}{$3^{e}$} & \multicolumn{2}{|l|}{$4^{e}$} & \\
\hline & $\mathrm{N}$ & $\%$ & $\mathrm{~N}$ & $\%$ & $\mathrm{~N}$ & $\%$ & $\mathrm{~N}$ & $\%$ & \\
\hline Ovines et Caprinés & 307 & 89,7 & 3838 & 84,7 & 5459 & 90,4 & 803 & 70,0 & 10407 \\
\hline Bovinés & 25 & 7,3 & 635 & 14,0 & 412 & 6.8 & 283 & 24,7 & 1355 \\
\hline Suinés & 2 & $<1$ & 30 & $<1$ & 124 & 2,0 & 29 & 2,5 & 185 \\
\hline Anrilopincs Oryginés & 8 & 2,3 & 9 & $<1$ & 16 & $<1$ & 19 & 1,6 & 52 \\
\hline Canidés & - & - & - & - & 9 & $<1$ & 5 & $<1$ & 14 \\
\hline Viverridés & - & - & 9 & $<1$ & I & $<1$ & 2 & $<1$ & 12 \\
\hline Léporidés & - & - & - & - & 1 & $<1$ & - & - & 1 \\
\hline Oiseaux & - & - & 1 & $<1$ & 3 & $<1$ & 3 & $<1$ & 7 \\
\hline Indéterminés & - & - & 6 & $<1$ & 8 & $<1$ & 3 & $<1$ & 17 \\
\hline TOTAUX & 342 & & 4528 & & 6033 & & 1147 & & 12050 \\
\hline
\end{tabular}

Tableau 1 : Répartition de la faune carnée consommée (nombre de restes osseux) dans la grotte Capéletti (Aurès, Algérie d'après P.L. Carter et E. Higgs, in Roubet 1979).

D'autres informations découlent de la maîtrise de cette domestication animale. Elles installent en effet l'élevage et la gestion des troupeaux au centre d'un projet fondamental et communautaire, au cœur d'un vécu novateur. Ce projet-moteur, vecteur de richesse et de surplus, dynamise cette première économie pastorale. Collective, planifiée, cette économie est passée d'un stade autarcique (première série d'occupation) à un stade ouvert sur l'échange, qui a accéléré le développement communautaire, socio-économique et culturel, favorisé la propagation des pasteurs et leur genre de vie à travers l'Atlas Saharien, pendant plus de deux mille ans. Restant à dessein fondamentalement pré-agricole, ce comportement choisi ne traduit pas l'ignorance de nouvelles pratiques de production végétale adoptées ailleurs, ni même l'incertitude des résultats, mais semble admettre l'inadéquation d'un nouveau projet de production végétale, entrepris dans des conditions peu favorables en altitude, des déplacements saisonniers et une certaine conception cyclique du temps (Ferré 1979).

9 Ce premier type de pastoralisme est donc resté pré-agricole comme l'attestent les végétaux issus des dépôts de la grotte Capelétti (Plu, Portères 1979). Ces carpo-restes 
résultent de collectes récurrentes et saisonnières. L'éventail des fruits massivement récoltés (arilles, glands, raisins) et consommés en grotte avant la fin de l'automne, laisse entrevoir des connaissances biogéographiques précises sur les potentialités renouvelées de cet environnement montagnard. Mais il est un fruit dont les propriétés et le goût ne ressemblent à aucun autre, qui fut particulièrement recherché. C'est le gland doux, non amer et un peu sucré, du chêne yeuse, variété ballote, si caractéristique de ce séjour montagnard (Portères 1979). A partir de récoltes automnales récurrentes j'ai pu souligner l'indirecte apparition, pour la première fois, de semoules (ou farines) obtenues à partir de leur transformation par broyage. Cette information est capitale; en effet, l'entrée de la première production de farine et de semoule, l'apparition des premières bouillies dans l'alimentation des pasteurs ne furent ni anecdotiques ni de même nature que d'autres faits de préhension. Cette substance s'est avérée si nutritive et appropriée (aux jeunes et aux individus âgés) qu'elle a installé plus qu'un goût, de nouveaux besoins, réclamant de plus grandes quantités de glands, entraînant la quête d'autres boisements de chênes yeuses de cette variété, qu'on ne retrouve qu'en milieu calcaire entre 400 et $1600 \mathrm{~m}$ d'altitude. Ce type spécial de production de farine, tirée de fruits sauvages broyés, me paraît nuancer le caractère pré-agricole de cette néolithisation. Les nouvelles préparations culinaires que le gland doux a enclenchées sont significatives de changements alimentaires, exigeant des récoltes spéciales, cycliquement programmées, nouvel objectif des pasteurs en montagne. Voilà pourquoi, sur le chemin ayant conduit les hommes à mettre en place en plaines des pratiques agricoles, j'estime que nous pourrions retenir cet exemple comme ayant pu prédisposer les pasteurs à chercher à découvrir sur d'autres montagnes environnantes de l'Atlas les mêmes ressources naturelles avant de chercher à s'investir en plaine dans un nouveau domaine agricole non maitrisé. Ces semoules de glands doux doivent donc être interprétées comme une solution de substitution aux céréales, opportune et typique des économies montagnardes, adoptées par d'autres populations néolithisées, méditerranéennes. Le stockage des glands en vue d'une consommation différée s'y ajoute comme étant une autre solution anticipatrice mise an place par les pasteurs de l'Aurès.

10 Le projet pastoral communautaire qui découle donc de l'analyse intégrative des biodocuments, sans qu'intervienne encore la dimension culturelle, ne s'est pas figé, n'a pas conduit les pasteurs à se replier, ni à vivre en autarcie. La gestion dynamique des troupeaux, les transhumances annuelles, entrâ̂nèrent l'éclatement et la dispersion des communautés. Cette indéniable accélération de la propagation en tandem du pastoralisme et de la glandée automnale caractérise la néolithisation atlasique en Algérie. Les transhumances ont favorisé l'établissement de relations sociales avec d'autres catégories de nomades. Des actes d'échanges attestent la mise en place d'un troc (Roubet 2001, 2003, 2010). C'est à la suite de ces considérations qu'un statut de berger fut perçu et reconnu. Reposant sur une production de la subsistance, suffisante et renouvelée, ce statut s'est imposé parce qu'il s'appuyait sur un capital humain et animal croissant, renouvelé et mobile, une cohésion comportementale du groupe, un vaste territoire et une résidence en altitude, critères conférant ensemble et au groupe tout entier, du pouvoir et un certain prestige. L'acte d'échange pratiqué avec d'autres partenaires, non pasteurs, chargés d'objets rares et exogènes, conduisit à une identification d'autres statuts et une reconnaissance réciproque des partenaires, et à l'attribution d'un statut de colporteurs, aux uns et d'un statut d'artisans spécialistes, 
aux autres (Roubet 2003). Un réseau social s'est ainsi esquissé. Ce modèle comportemental aurasien a pu être étendu au massif des Nemencha.

\section{- Massif des Némencha}

11 Dans les Nemencha*, les données chronologiques, fauniques, et culturelles sont issues de deux Abris du Damous el-Ahmar, localisés dans le Kef el-Ahmar (1300m d'alt.) du Djebel Anoual, près de Tébessa (Roubet 1968 ; Camps-Fabrer EB XIV, p. 2194-2203). La domestication animale (ovins-caprins) a été établie par P. L. Cater (1979) qui s'appuyait sur la faune de la grotte Capéletti. Cependant ces restes osseux n'ont pas fait l'objet de décomptes. Il faut en outre rappeler les incertitudes (localisation, profondeur) qui affectent la documentation issue des premières fouilles de M. Latapie en 1912, accompagnées pourtant de Notes. D'autres travaux limités ont été pratiqués, il s'agit de deux sondages l'un effectué en 1964 (Roubet 1968) et l'autre en 1973 par E. Poty (Roubet 1979). Deux datations ont été obtenues à partir du sondage de C. Roubet :

Alg11 : 050-150 cm : coquille d'œuf d'autruche $5400 \pm 190$ BP soit $3450 \pm 190 \mathrm{BC}$ ou 4676-3794 cal BC.

Alg10 : 150-160 cm : hélix : $5720 \pm 195$ BP soit 3770 195 BC

Les données anthropologiques, qui manquaient dans l'Aurès, proviennent de plusieurs inhumations découvertes en 1912 et en 1973 ; elles montrent que ces communautés de pasteurs étaient d'ascendance Mechtoïde. Leurs séjours saisonniers (printempsautomne) au Damous avec leurs troupeaux de moutons, chèvres et bovins sont au moins contemporains de la troisième série d'occupation de Capéletti. Retenons encore une information précisant un comportement de vigilance des pasteurs passant par la gravure, utilisée de façon éducative. Sur un bloc calcaire H. Camps-Fabrer et C. Roubet ont découvert la gravure rapide de trois animaux identifiables qui établit un rapport direct de ces pasteurs avec leur troupeau. Son message a le mérite de souligner que la vigilance des troupeaux s'imposait à tous les membres. La scène représente deux renards vus de profil qui regardent sous leur museau la maigre dépouille qui subsiste de leur larcin déchiqueté. Nul doute qu'il s'agisse de l'agneau qu'ils viennent de capturer et dévorer (Roubet 2001, 2010). Dans ce contexte pastoral qui fit vivre et s'installer les bergers et leurs troupeaux au Damous el-Ahmar (Kef el-Ahmar du Djebel Anoual), il est évident que l'approche de tout carnivore était à redouter. Avec cette esquisse, c'est une leçon de prudence que les pasteurs souhaitèrent donner à leurs jeunes bergers insouciants. En la gravant à la hâte, avec le souci de l'exemple, un pasteur a retenu la silhouette de deux renards impudents et rassasiés et l'indéchiffrable dépouille d'un agneau perdu. Voilà comment s'est exprimée par le croquis pris sur le vif, une forme d'interactivité éducative entre adultes et jeunes bergers. L'esquisse réaliste de deux renards repus et de leur larcin déchiqueté n'est pas celle d'une œuvre d'art, sa fonction a donc été de délivrer un message de prudence soulignant la perte d'une jeune bête. Cette œuvre est unique dans l'Atlas saharien. 


\section{Représentations graphiques et symboliques de la domestication animale dans l'Atlas saharien de l'Algérie}

13 Comme l'avait souligné G. Camps (EB IX, p. 1417-1433) à propos des nombreuses gravures du bélier à sphéroïde, à travers l'Atlas saharien, le thème de «l'ovin paré d'une coiffure globuleuse de forme sphérique et de divers attributs " contribue à accroître nos connaissances sur l'animal domestique, dans une posture symbolique.

Ces représentations sont toutes étrangères à la notion de troupeau, chaque artiste place l'observateur devant un seul animal élevé au sublime. Toute gravure est une sublimation. Une soixantaine d'œuvres est disséminée depuis les Némencha jusqu'aux Ksour, toutes gravées (pas de peintures) selon les mêmes canons, donnant au seul animal vivant, figé que l'on voit de profil, une silhouette constante et typique identifiable : souvent celle d'un jeune mâle, qui appartient à l'espèce à poil ras, Ovis longipes Fitzinger. On peut admettre que cette espèce très ancienne est celle qui s'est répandue domestiquée en région atlasique lors de la néolithisation pastorale. Lorsqu'on tente de comparer les restes osseux issus de dépôts aux gravures, on constate que les traits anatomiques de la tête ovine, si caractéristiques sur les gravures, avec un museau camus et de jeunes chevilles osseuses, ne sont pas aussi accusés sur les pièces des dépôts de la grotte Capéletti. On se heurte ensuite à l'interprétation symbolique de la tête par les différents artistes (gravure soignée et enrichie de détails ou gravure sommaire), et dans les fouilles, à l'absence de témoins de parure (en os, en bois, ou fait de tressages), qui furent disposés sur la tête (sphéroïde), autour du cou (collier), et sur l'échine (une croupière en peau). Le rapport d'identification de ces œuvres aux témoins paléontologiques reste donc délicat à établir. Chaque œuvre nous renvoie au talent du graveur. Un excellent document (Bou Alem) a été photographié par R. Vaufrey (1939).

Quoi qu'il en soit, et en dépit des lacunes chronologiques, que notre collègue Malika Hachid tente de combler (campagne de prélèvements en 2008), nous pouvons résumer ainsi les informations pariétales, relatives à cette représentation, évocatrice de la néolithisation :

- Aucun troupeau ovin, caprin, bovin n'a été représenté dans l'Atlas saharien algérien ;

- Aucune scène pastorale n'évoque le rapport direct et intime entre l'animal domestique et l'homme, notamment lors de l'agnelage et de la traite ;

-Aucune scène ne révèle le sacrifice du mouton, avec ou sans les officiants et leurs instruments ;

- L'ovin orné est si précisément embelli qu'un rituel se trouve indirectement exprimé pour informer l'assistance de l'événement qui va s'accomplir et que chacun pourra observer en plein air (G. Camps, p. 1432),

- La tête intentionnellement relevée de l'ovin (quel que soit le poids de la parure) donne de la prestance à l'animal et inscrit la cérémonie dans une sublimation ayant une valeur sociale de communion. A la manière d'une "affiche ", la gravure est donc annonciatrice d'un événement.

- Le contraste graphique entre l'homme - lorsqu'il est présent - et l'animal paré a pour but de le confirmer en réduisant l'importance et le rôle de l'humain (gravure stylisée, de petites dimensions, avec ou sans instrument, ni traits distinctifs, comme anonyme, pour ne pas identifier le sacrificateur), alors que le bélier choisi est un animal jeune, viril, de belle taille, 
au beau cornage, sélectionné et traité pour être vu, à travers le poil lisse et peigné, et par la parure déposée, comme un animal majestueux destiné au sacrifice. que le thème majeur de l'offrande animale dont s'imprègnent les cultures du bassin méditerranéen plonge ses racines dans cette forte expression collective du sacré qui dût entourer ces cérémonies néolithiques?

Signalons enfin qu'en Tunisie atlasique, dans la Dorsale* Tunisienne, un nouveau thème, celui de l'ovin entravé, apparait sans décor sur la tête. Il ne s'agit donc plus du «bélier à sphéroïde» de l'Algérie pré-saharienne. L'ovin n'est pas ici isolé. La découverte rapportée par S. Yahia-Achèche (2007) est celle d'une gravure nette d'ovin non paré, présente dans le Djebel Ousselat*, dans la station de Ghorfat-er-Rmada. Ce site ( $715 \mathrm{~m}$ d'altitude) occupe la rive droite de l'oued Ellouz. Le bestiaire entourant cette œuvre est composé d'une grande faune sauvage. Sur un panneau s'observent le rhinocéros et l'antilope bubale, traitées dans un style « bubalin naturaliste ».

«Sur un autre panneau, des animaux de la faune sauvage côtoient des espèces domestiquées (caprinés) ; des personnages et des téranthropes (mi-hommes, mianimaux) sont aussi représentés en étroite relation avec les animaux. Les figures s'imbriquent les unes aux autres. Le bâton que portent tous les personnages pourrait être le symbole d'un mode de vie pastorale, de même que l'entrave qui est placée au cou de certains bovidés ».

La néolithisation pastorale atlasique est un thème de recherche capital, peu développé, qui livre déjà des informations décisives bien qu'éparses encore, qu'il conviendra de développer et de traiter en fonction de l'objectif économique et symbolique que les pasteurs poursuivirent. Cependant si ce tout premier projet que l'élevage permit d'entrevoir et de structurer en montagne, connut un tel succès et se propagea si bien, c'est aussi parce qu'il resta greffé à d'ancestrales traditions structurantes, atténuées. En se dispersant à travers les chaînons de l'Atlas, ces communautés montagnardes maintinrent certains traits culturels qui nous permettent de mailler et valoriser un relationnel insoupçonné, d'entrevoir une communication fondée sur l'échange. Toutes ces communautés restèrent fixées en altitude, elles enracinèrent des comportements fondamentaux que conservent les populations rurales berbères.

\section{BIBLIOGRAPHIE}

CARTER P.-L., et HIGGS E. S., 1979 - « A study of the faunal remains from La grotte Capéletti du Khanguet Si Mohamed Tahar (Aurès, Algérie) », in Roubet C., Economie pastorale, préagricole, en Algérie orientale. Le Néolithique de tradition capsienne. Exemple : l'Aurès, Paris, CNRS, p. 411-414.

FERRÉ B., 1979 - « Etude sur l'ensoleillement de l'entrée de la grotte Capéletti », in Roubet C., Economie pastorale, préagricole, en Algérie orientale. Le Néolithique de tradition capsienne. Exemple: l'Aurès, Paris, CNRS, p. 521-546. 
PLU A. et PORTÈRES R., 1979 - « Identification des macro-restes végétaux de la grotte Capéletti », in Roubet C., Economie pastorale, préagricole, en Algérie orientale. Le Néolithique de tradition capsienne. Exemple: l'Aurès, Paris, CNRS, p. 426-448.

PORTÈRES R., 1979 - « Présence et utilisation des végétaux identifiés dans la grotte Capéletti », in Roubet C., Economie pastorale, préagricole, en Algérie orientale. Le Néolithique de tradition capsienne. Exemple: l'Aurès, Paris, CNRS, Paris, p. 439-448.

ROUBET C., 1968 - Le gisement du Damous el Ahmar et sa place dans le Néolithique de tradition capsienne. Travaux du CRAPE, Paris, AMG., 144 p.

ROUBET C., 1979 - Economie pastorale préagricole en Algérie orientale : le Néolithique de tradition capsienne. Exemple de l'Aurès, Paris, CNRS (Etudes d'Antiquités Africaines), 595 p.

ROUBET C. et Carter P.-L. 1984 - « Origine de la transhumance ovine dans le Maghreb oriental », in The Deya Conference of Prehistory : Early settlement in Western Mediterranean Islands and the peripheral areas, Ed. by W. H. Waldren and coll. B.A.R., International Series, $n^{\circ} 229$, p. 239-248.

ROUBET C., 1985 - « Transhumance ovine dans l'Aurès depuis le Ve millénaire B.C. », Studi di Paletnologia in onore di Salvato M. Puglisi. Ed. by M. Liverani, A. Palmieri, R. Peroni, Università di Roma, La Sapienza, 1985, p. 421-423.

ROUBET C., 2001a - « Le Néolithique de Tradition Capsienne sensu strict », Encyclopedia of Prehistory. Volume 1: Africa, 10 entrées, in Outlines of Archaeological Traditions. The Human Relations Area Files, Ed. by P.-N. Peregrine and M. Ember, Kluwer Academic, Plenum Publishers, p. 197-219. ROUBET C., 2003 - «Statut de berger » des communautés atlasiques néolithisées, du Maghreb oriental », L'Anthropologie, t. 107, p. 393-442.

ROUBET C., 2006 - « Pastoralisme et ruralité néolithiques dans l'Aurès, avec la grotte Capéletti, entre $7500 \mathrm{cal}$ BP et $5400 \mathrm{cal} \mathrm{BP} »$, Aouras, n 3, p.19-35.

ROUBET C., 2011 - « Statut social des pasteurs néolithiques des Némencha : Objets de prestige », Aouras, $n^{\circ}$ 6, p. 39-55.

ROUBET C., HACHI S. et KHERBOUCHE F., 2010b - « Vivre ensemble depuis 20000 ans au moins en Afrique du Nord : expression d'une communication ", Nouveaux Mélanges offerts à Paulette GalandPernet et Lionel Galand, à l'occasion de leur $90^{e}$ anniversaire. A. Mettouchi ed., Cologne, Rüdiger Köppe Verlag, p. 1-30.

VAUfReY R., 1939 - L'Art rupestre Nord-Africain. Paris, Masson (Archives de l'Inst. de Paléontologie Humaine, t. XX), $128 \mathrm{p}$.

YAHIA-ACHACHE S., 2007 - L'art rupestre de Tunisie. Inventaire et Analyse. Ses relations avec les foyers artistiques de l'Algérie et la Libye voisines. Thèse de Doctorat, Paris 1-Panthéon-Sorbonne, 412 p.

\section{INDEX}

Mots-clés : Algérie, Néolithique 